\title{
Assessment of knowledge, skill and attitude of oncology nurses in chemotherapy administration in tertiary hospital Pakistan
}

\author{
Najma Khan ${ }^{1}$, Khurshid Zulfiqar Ali Khowaja ${ }^{2}$, Tazeen Saeed Ali $^{3}$ \\ ${ }^{1}$ Army Hospital, Rawalpindi, Pakistan \\ ${ }^{2}$ School of Nursing, Aga Khan University, Karachi, Pakistan \\ ${ }^{3}$ Department of Community Health Sciences, Aga Khan University, Karachi, Pakistan \\ Email: ummadk@yahoo.com, khurshid.khowaja@alnoorhospital.ae, tazeen.ali@aku.edu
}

Received 29 December 2011; revised 8 February 2012; accepted 22 February 2012

\begin{abstract}
Objective: To measure the levels of nurse's knowledge and attitude after the conduct of education session regarding chemotherapy administration and management. Methodology: This research study was conducted at two oncology units of tertiary Hospital Rawalpindi. A pre-post test intervention study design was used on 35 nurses by using Verity's tools. Results: The mean scores of knowledge were calculated by Cochran's Q test showed that knowledge scores have significantly increased with "educational training" (p value $<\mathbf{0 . 0 0 1}$ ). The difference in the attitude of the nurses was not found to be statistically significant in repeated measure of ANOVA. Conclusion: The results show that knowledge is the weakest component and attitude is strongest component of oncology nurses competencies in chemotherapy administration.
\end{abstract}

Keywords: Oncology Nurse; Knowledge; Attitude; Chemotherapy Administration; Crush Programme

\section{INTRODUCTION}

Cancer is leading cause of death worldwide, whereby more than 10 million people are diagnosed with cancer and 6 million deaths take place annually [1]. It has been estimated that there will be 15 million new cases every year by 2020 [2]. The disease burden is much higher in Pakistan than developed countries [3]. The current data on cancer incidence is not available due to unavailability of regular cancer registry system at national level in Pakistan, according to last available figures of 1998 [4], the rate of incidence for all cancers is $132.4 / 100,000$ for males and 133.0/100,000 for females [5].

Pakistan is lacking in financial and human resources to manage cancer especially in advanced stages. In health care field, there is a dearth of nurses especially oncology certified nurses, when there is an increased patient volume giving the nurse patient ration of 1:313. This makes nurses overworked and further due to limited resources, they do not have enough opportunity to enhance knowledge and skills in their specialty [6,7].

The main role of nurses in the field of oncology is chemotherapy administration which is sensitive domain in oncology nursing where little negligence or mistake may lead to adverse consequences for patients, staff and environment $[8,9]$. Literature reveals that, medication errors in chemotherapy is a common (44\%) [10] incidence due to lack of specific knowledge and training of the staff in chemotherapy, prescription, preparation and administration [11,12]. This lacking of knowledge and training of the staff leads to fatal incidences such as over dose of chemotherapy, wrong calculations of drugs, wrong route of transfusion which sometimes result in patient's death. Therefore studies have been conducted in past to evaluate the knowledge and attitude of nurses in different specialties [13], and proved that nurses; knowledge can be enhanced with the help of educational programmes [14-16].

Nurses caring for patients receiving chemotherapy require specialized knowledge in order to ensure safety for both patients life and for their own safety of the jobs. Many nurses have been fired from their job due to medication errors.

Chemotherapy is one of the most commonly prescribed cancer treatment modality [17] which has complex treatment regimes with advanced technological devices. Nurses who used to administer chemotherapy need to update their clinical and theoretical knowledge including drug calculations, appropriate dilutions, identification of antidotes of cytotoxic drugs, and management of side effects particularly in case of extravasations, [18] providing support and advocacy for patients experienc- 
ing profound psychosocial and existential distress [19]. Along with technical expertise for ensuring safe administration of treatment, nurses have to demonstrate a high level positive attitude to help cancer patients in their psychological issues due to chemotherapy.

Attitude is the underlying way we think, feel, act, and react to the world around us [20]. Attitude includes manner and the mind set in which one approaches care; it encompasses the essence of caring, interpersonal communication, individual uniqueness, and respect for humanity.

It is observed that in majority of the oncology units in Pakistan, chemotherapy is being prepared, administered by oncology nurses along with the management of its side effects if patient have. So nurses are being responsible for complete the whole process of chemotherapy. Hence, there is a great need to assess the knowledge, skill and attitude of oncology nurses in Pakistan to ensure the safe care in chemotherapy administration of cancer patients. Therefore, this study was conducted to assess the oncology nurses' existing level of knowledge, skills and attitude regarding chemotherapy administration.

\section{METHODOLOGY}

Single group pre-test post-test study design was conducted in two oncology units of tertiary hospital, Pakistan between June and August 2010. Both oncology units cater all kind of cancer patients at the inpatients and outpatients departments for adult and pediatric cancer patients. The nurse-patient ratio was in unit one is 1:30 in unit two 1:8.

The targeted population comprised of registered enrolled nurses currently administering chemotherapy and working in oncology units with minimum work experience of six months.

The universal sampling technique was employed. The assessment of participants' knowledge, skills and attitude regarding chemotherapy was done through questionnaire with, MCQs and skills were observed through checklist. On Average it took 45 minutes to complete the questionnaire and $1-1.30$ hours to observe their skills.

We invited all the available 39 nurses for the study who meet the inclusion criteria with $95 \%$ of response rate $(n=35 / 39,95 \%)$, however four out of them had excluded from the study due to less working experience and having pregancy. So, finally 35 participants were enrolled in this study. The tool for knowledge and attitude assessment for chemotherapy administration was used. The face, content, construct and criterion validity and reliability of questionnaire were established [21].

Knowledge assessment tool included 11 questions with 4 relevant options. The highest possible score would be 11. Questions focused on safety measures for patients and nurses, knowledge about the preparation, dilution, and administration of chemotherapy, management of side effects, and emergency treatment of the patient.

The attitude scale included a 21 items statement on a 4 point Liker scale, the highest possible score, indicating a positive attitude, would be 84 . The attitude scale included both positive and negative item statements. Positive attitude statement were scored as $4=$ strongly agree, 3 = agree, 2 = disagree, 1 = strongly disagree, conversely negative attitude statements were scored $1=$ strongly agree, 2 = agree, 3 = disagree, 4 = strongly disagree.

In addition to assessing the knowledge of chemotherapy administration, demographic characteristics (age, professional qualification, oncology qualification,) and information regarding working experience as chemo nurse was recorded.

Study approval was obtained from the Ethical Review Committee of the Aga Khan University. Permission was sought hospital administration to conduct the study in the respective units.

All data were entered in the SPSS version 17; descriptive and inferential statistics were used to analyze the data. The data were analyzed by comparing of the pre and post knowledge of nurses by using of nonparametric alternatives method of Cochran Q test. The Cochran Q test is used when variables are measured in terms of categories or proportions, e.g., "correct" vs. "incorrect". For analysis of MCQs, of knowledge question, one point was assigned for each correct response. The incorrect responses were assigned a score 0 . The total scores were obtained by adding scores for all the correct responses, at the end of the test aggregated scores were used for statistical analysis. The difference between the overall score pre-test, post-test one and post-test two was analyzed through repeated measure of ANOVA, which is specialized test to examine the difference in the means of more than two groups.

\section{RESULTS}

\subsection{Demographics}

All the nurses were female, with the mean age of being 24.1 and $S D \pm 2.0$ years. The majority of nurses 24 (68\%) were between 20 - 40 years and 11 (31\%) of them were more than 40 years of age. The data on professional qualifications showed 28 (80\%) were undergraduates, and 7 (20\%) had graduated in nursing education from the army nursing schools. Out of 28 (undergraduate) nurses, 11(33\%) had received oncology course certification from the Army school of Nursing and only 2 (5\%) nurses had received bone marrow certification from Canadian institute.

The information regarding participants' working ex- 
perience shows that the majority 18 (51\%) had more than 10 years of working experience as a general nurse, while 16 (46\%) had less than 5 years of work experience as a chemo nurse, indicating that the majority of the study participants 19 (54\%) had less work experience as chemo nurse (see Table 1). Results of the study showed that all participants were female, majority were undergraduates and have less working experience as chemo nurse, and only one third (30\%) were oncology trained.

\subsection{Knowledge}

The results highlighted that only 3\% participants were

Table 1. Personal characteristics of female nurses working in Army hospital, Rawalpindi, Pakistan $(\mathrm{n}=35)$.

\begin{tabular}{|c|c|c|}
\hline Variables & $\mathbf{n}$ & $\%$ \\
\hline \multicolumn{3}{|l|}{ Age } \\
\hline 20 to $40 \mathrm{yrs}$ & 24 & 69 \\
\hline More than 40 yrs & 11 & 31 \\
\hline \multicolumn{3}{|l|}{ General Qualification } \\
\hline Metric (ten years of education) & 6 & 17 \\
\hline FA (twelve years of education) & 14 & 40 \\
\hline BSc (fourteen years of education) & 13 & 37 \\
\hline MSc (sixteen years of education) & 1 & 3 \\
\hline \multicolumn{3}{|l|}{ Professional Qualification } \\
\hline Undergraduate & 28 & 80 \\
\hline Graduate & 7 & 31 \\
\hline \multicolumn{3}{|l|}{ Special Course } \\
\hline Oncology & 11 & 31 \\
\hline BMT Nursing & 2 & 6 \\
\hline \multicolumn{3}{|l|}{ Years of Experience as Nurse } \\
\hline $1-4$ yrs & 7 & 20 \\
\hline $5-9$ yrs & 10 & 29 \\
\hline 10 yrs and Above & 18 & 51 \\
\hline \multicolumn{3}{|c|}{ Years of Experience Caring Cancer Patient } \\
\hline $1-4$ yrs & 25 & 29 \\
\hline $5-9$ yrs & 6 & 43 \\
\hline 10 and Above & 4 & 11 \\
\hline \multicolumn{3}{|c|}{ Years of Experience of Chemotherapy Administration } \\
\hline $1-4$ yrs & 16 & 46 \\
\hline $5-9$ yrs & 9 & 26 \\
\hline 10 yrs and Above & 10 & 30 \\
\hline
\end{tabular}

able to get $80 \%$ scores and $54.3 \%$ able to get $60 \%$ scores in chemotherapy knowledge. Out of a total of 11 items of knowledge related to chemotherapy administration, in seven items where nurses got more than $60 \%$ scores including a nurse's immediate action on chemotherapy splashes (88.6\%), a small spill is defined (80\%), group of biologic agents must be handled as cytotoxic agents? (94.3), family teaching about contaminated linens (65.7), chemotherapy splashes into one eye (80\%), and regarding information for a pregnant chemo nurse (71.4) (see Table 2).

\subsection{Attitude}

The results of the study illustrate that the participants' attitude regarding chemotherapy administration and management was mid way, the mean value of the participants attitude was 58.2, which was higher than the other two components (knowledge and skills).

Participants demonstrated their negative attitude towards chemotherapy administration by agreeing with negative statements. The negative attitude section shows that although out of 12 items, only five items displayed the negative attitude of the participants. The item (a) "Giving chemotherapy to patients makes me feel guilty" $75 \%$ were participants agreed with this statement. Similarly, for item (b) "I know that all precautions are taken to ensure that I am not at risk from chemotherapy” 63\% nurses were disagree with this positive statement, for item (c) "I try to avoid patients that are experiencing bad side effects from chemotherapy" 86\% nurses were agree with this statement, for item (d) "chemotherapy causes more harm than good" 58\% nurses were agree with this statement and for item (e) "It is easy to inform patients about their treatment but harder to help them emotionally” 75\% nurses demonstrated their negative attitude by agreeing with this statements (See Table 3).

The positive attitude section shows that out of 9 items, only fur items displayed the significant change in the correct attitude of the participants after the educational sessions. These correct attitude items include: item (a) "I always feel that I have supported patients during their chemotherapy treatment in which 74\% nurses agree with this statement. Similarly, for item (b) "I know that when I administer chemotherapy patients have understood as much as they wish to about their treatment" $83 \%$ nurses were agree with this statement for item (c) "When patients present complications caused by chemotherapy, I feel confident in my competency to handle them" "89\% nurses and for item (d)" Intravenous chemotherapy transfusion is different than transfusion of routine antibiotics" 54\% nurses agreed with this statement which showed a high positive attitude towards chemotherapy administration (Table 4). 
Table 2. Individual items and respective scores assessing knowledge of oncology nurses in pre, post 1, and post 2 with percentage of correct responses and p value.

\begin{tabular}{lcccc}
\hline \multicolumn{1}{c}{ Correct Knowledge } & PRE n (\%) & Post 1 n (\%) & Post 2 n (\%) & p-Value Cochranıs Q \\
\hline Teratogenicity of hazardous drugs & $13(38)$ & $28(80)$ & $20(57)$ & 0.002 \\
Safety measure during bladder instillation & $8(23)$ & $20(57)$ & $16(46)$ & 0.024 \\
Precautions if cytotoxic drug splashes on skin & $31(88)$ & $35(100)$ & $35(100)$ & 0.018 \\
Practices regarding releasing a hazardous drug while priming IV tubing & $11(32)$ & $23(66)$ & $20(57)$ & 0.018 \\
Definition of spill & $28(80)$ & $33(94)$ & $35(100)$ & 0.013 \\
$\begin{array}{l}\text { Biologic agents to be handled according to cytotoxic guidelines } \\
\text { Handling of contaminated linens of post chemo urinary incontinence pt at }\end{array}$ & $33(94)$ & $18(51)$ & $28(80)$ & $<0.001$ \\
home & $23(65)$ & $32(91)$ & $27(77)$ & 0.055 \\
Education of nurses for chemotherapy administration & $23(66)$ & $23(66)$ & $32(91)$ & 0.011 \\
Nurse's first action if cytotoxic drug splashes into eye? & $28(80)$ & $35(100)$ & $35(100)$ & 0.005 \\
Competency evaluation of nurses & $11(31)$ & $34(97)$ & $31(88)$ & $<0.001$ \\
$\begin{array}{l}\text { Essential information about chemotherapy administration for pregnant } \\
\text { nurse }\end{array}$ & $25(71)$ & $34(97)$ & $35(100)$ & $<0.001$ \\
\hline
\end{tabular}

Table 3. Negative attitude.

\begin{tabular}{|c|c|c|c|}
\hline \multirow{2}{*}{ Negative Attitude } & \multicolumn{3}{|c|}{ Strongly Agree/Agree } \\
\hline & Pre-test & Post test 1 & Post test 2 \\
\hline I did not feel confident in my competency to handle chemotherapy emergencies & $42 \%$ & $46 \%$ & $23 \%$ \\
\hline I try to avoid patients that are experiencing bad side effects from chemotherapy & $86 \%$ & $92 \%$ & $89 \%$ \\
\hline Giving chemotherapy to patients makes me feel guilty. & $75 \%$ & $80 \%$ & $88 \%$ \\
\hline It is easy to inform patients about their treatment but harder to help them emotionally & $75 \%$ & $86 \%$ & $83 \%$ \\
\hline Giving chemotherapy impedes communication with patients. & $94 \%$ & $77 \%$ & $85 \%$ \\
\hline I know that all precautions are taken to ensure that I am not at risk from chemotherapy & $37 \%$ & $28 \%$ & $20 \%$ \\
\hline
\end{tabular}

Table 4. Positive attitude.

\begin{tabular}{|c|c|c|c|}
\hline \multirow{2}{*}{ Positive Attitude } & \multicolumn{3}{|c|}{ Strongly Agree/Agree } \\
\hline & Pre-test & Post test 1 & Post test 2 \\
\hline I always feel that I have supported patients during their chemotherapy treatment & $74 \%$ & $97 \%$ & $100 \%$ \\
\hline $\begin{array}{l}\text { I know that when I administer chemotherapy patients have understood as much as they } \\
\text { wish to know about their treatment }\end{array}$ & $83 \%$ & $89 \%$ & $94 \%$ \\
\hline I know enough about chemotherapy to answer patients questions adequately & $86 \%$ & $78 \%$ & $68 \%$ \\
\hline I feel confident in my competency to handle complications caused by chemotherapy & $94 \%$ & $89 \%$ & $89 \%$ \\
\hline
\end{tabular}

\subsection{Skills}

The skill of the participants tested at three stages such as pre administration, during administration, and post administration. Each skill item was marked YES or NO and analyzed by using the Cochran's Q test.

Pre Administration Stage: The significant items in skill section where participants got more than $80 \%$ marks in pre administration stage including verify MD order for dosage of drugs, review checklist, obtain drugs from pharmacy \& verify MD with orders, regarding skills necessary for during administration stage including wash hands prior to administration, prepare work area for administration, primes chemotherapy, check potency of intravenous line just prior to initiation of therapy, administer chemotherapy as per order, monitor for adverse re- 
action and on completion of therapy flush with compatible solution and skills important for post administration stage including document procedure in patient record and monitor patient for post side effect and give antiemtics.

All these nursing procedures are commonly learnt in the basic nursing course and nurses are well aware of their use in their practices.

The overall finding of the skills indicated that out of 61 skills items in all three stages, the twelve items where participants got more than $80 \%$ marks and 8 items where they got 50\% marks (see Figure 1).

\section{DISCUSSION}

\subsection{Knowledge}

The overall finding of the study indicated that the participants have poor knowledge and skills, however their attitude is good. This finding is important for nursing care to cancer patients because if oncology nurses did not have advance knowledge and not competent in their skills will be considered as unsafe for providing chemotherapy administration to cancer patients and chances for medication errors can be high as various studies in past already highlighted these points [22,23]. Nurses' awareness and knowledge about the handling of cytotoxic drugs remains a concern linked to improvement in safety standards. The higher the nurses' knowledge the more they use the safety measures in their practices. Literature reports that there is a gap between the nurses' knowledge and their actual behavior with respect to the use of protective measures [24,25].

\subsection{Skills}

Chemotherapy preparation, administration, management of side effects, and related emergencies are key skills in oncology practice. Twenty three actions were observed while the nurses prepared, mixed, and administered chemotherapy in their work surroundings.

The overall findings of the study indicated that in majority of skill items nurses have poor performances in all

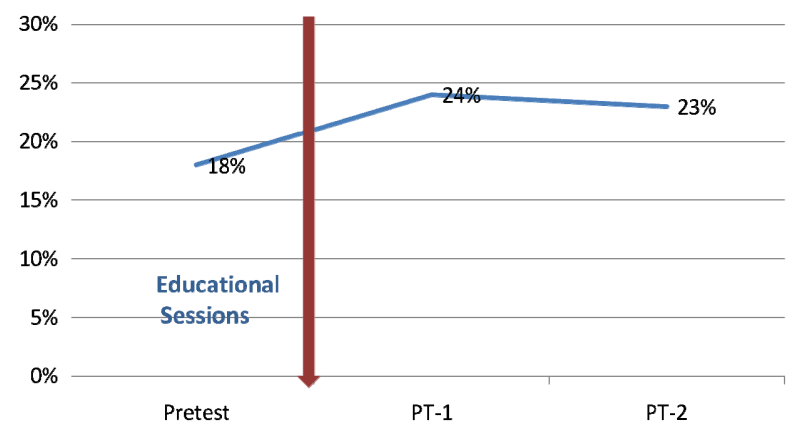

Figure 1. Mean scores of skills of chemotherapy administration before and after the educational sessions. three stages including pre administration, during administration and post administration of chemotherapy. However, during the skill observation process, the researcher observed that the study participants were not following the international standards for chemotherapy preparation and administration. The available guidelines had many gaps as per international standards and were not being properly practiced at the clinical setting. This finding is in line with Butt's (2008) study in the same setting, where she found that chemotherapy preparation and administration was not according to international standards, and recommends that chemotherapy should be prepared by trained pharmacist and administered only by trained nursing staff.

The current study identified that nursing skills were weak at all three stages including pre administration, during administration and post administration of chemotherapy. The nurses' skills were poor in all three stages in different skills items. In the pre administration stage, these included item (a) “check patient's allergies”, item (b) "calculate dosage based on BSA", item (c) "verify dosages with RN", and item (d) "check drugs \& identify modifiers with RN witness”. In the during administration stage, the item include: Item (a) "check patency of cannula in every shift until completion of therapy” and item (b) "identify patient drug modifier". In the post administration stage, there was one item (a) "disposes of cytotoxic agents”.

The justification given by them for their weak skills in the above mentioned areas was high work load, understaffing, and high patient volume. Similarly, they scored low in a number of items in the pre-test because these areas required comprehensive skills related to oncology clinical expertise, not routine skills for which they have been trained. They further explained that the assessment of a patient's allergies and measurement of a patient's BSA is the job responsibility of the oncology resident; therefore they assumed that it was not their responsibility. Moreover, they felt that they were not competent to do so because often the residents carried out the process again even if the nurses already done it.

In response to need for two nurses checked chemotherapy medication, the study participants responded that most of the time, one nurse was on duty in each shift; therefore, it was difficult to verify drugs with other registered nurse (RN) as witness of medication. Literature supports that verification of cytotoxic drugs with other nurses helps reduce medication errors in chemotherapy preparation, as medication errors commonly occur if cross checks are not done regularly [26]. The current study highlighted that due to understaffing or lack of knowledge the nurses were not checking patency of the intravenous cannula of each patient regularly. Checking the patency of the intravenous cannula prior to the ad- 
ministration of chemotherapy is a highly recommend practice in oncology nursing because most of the vesicant infusions are being transfused as chemotherapy and the vesicant group of drug are more potent, they are more likely to cause extravasations than other drugs. This negligence (not frequent checking the intravenous (I/V) cannula) by the nursing staff may cause severe emergency (extravasations) in an oncology setting. The oncology literature emphasizes the importance of regular checking of patency of intravenous cannula, as it is one of the main factors that can for prevent the extravasations of cytotoxic drugs [27]. In addition, the researcher observed that in spite of the fact, only a few nurses were post graduates they got high scores in safe I/V cannulation techniques. This was contrary to other studies [27] in which nurses' with-graduation (BScN) were found to be more competent than nurses with good experience in general nursing in ratings related to peripheral intravenous cannulation. Some studies proved that graduate level qualification was a significant correct predictor, and the years of experience in general nursing were a significant incorrect predictor with respect to the overall ratings of peripheral intravenous cannulation. In contrary, current study showed that the years of experience of nurses were a correct predictor in the assessment of safety cannulation techniques, as against the graduate level education.

\subsection{Attitude}

The attitude of the oncology nurses towards chemotherapy administration was assessed through the questionnaire.

In the negative attitude, nurses got higher scores by agreeing with negative statements, thus showing their negative attitude towards chemotherapy, including: "giving chemotherapy impedes communication with patients", "I try to avoid patients that are experiencing bad side effects from chemotherapy", "giving chemotherapy to patients makes me feel guilty", "it is easy to inform patients about their treatment but harder to help them emotionally" and "patients should always be informed about their treatment".

As is obvious from the result, the majority of the nurses were not oncology trained and had limited knowledge about the use and importance of cytotoxic drugs and the management of their physical and psychological side effects experienced by cancer patients. Therefore, may have developed some negative attitude towards chemotherapy administration. In consistent with my findings other studies [27] found that the nurses were inadequately prepared to care for cancer patients and consequently, held negative views about the disease and its treatment.
This is contrary to the other study [9] showed few nurses had a feeling of guilt and distress when they saw patients with complication; moreover, they did not avoid them. This study shows that nurses tried to avoid those patients who developed any complication caused by chemotherapy. This shows that the participants of the current study may have had less confidence, in their ability to manage complications, although they admitted that they were confident in this particular area. It has been proposed in previous research studies that although there is a possible correlation between knowledge and preventive behavior, the interaction is low (the level of knowledge does not greatly influence preventive behavior in practice).

The current study was the first of its kind in Pakistan in which effectiveness of educational sessions was assessed on the nurses' knowledge, and attitude. The educational session was found to be effective in improving the knowledge of nurses, however there was no significant change in their attitudes. This study would be a contribution to evidence-based literature in the Pakistani context. Despite of the narrow scope of the study and the various limitations, the results of current study may be assumed to be an important addition to the existing body of knowledge, especially in Pakistani context as no previous research within oncology nursing population, has been conducted in this field.

\section{CONCLUSION}

We recommend more educational session for the nurses to be able to bring in competency in nursing practices in chemotherapy administration.

\section{REFERENCES}

[1] WHO (2003) WHO’s fight against cancer: Strategies that prevent, cure and care.

[2] Horton, J. (2006) Breast cancer in 2020: What can we expect? Cancer Detection and Prevention, 30, 109-110. doi:10.1016/j.cdp.2006.03.003

[3] Ali, T.S. and Baig, S. (2006) Evaluation of a cancer awareness campaign: Experience with a selected population in Karachi. Asian Pacific Journal of Cancer Prevention, 7, 391-395.

[4] Ahmed, F., Mahmud, S., Hatcher, J. and Khan, S.M. (2006) Breast cancer risk factor knowledge among nurses in teaching hospitals of Karachi, Pakistan: A cross-sectional study. BMC Nursing, 1-7. http://www.biomedcentral.com/1472-6955/5/6

[5] Hameed, S., Hussain, A., Javed, N. and Subhani, H. (2009) Analysis of the Prevalence of cancer in Faisalabad: A single centre study. Asia-Pacific Microwave Conference, 3, 114-118.

[6] Kumar, S., Shaikh, A.J., Khalid, S. and Masood, N. (2010) 
Influence of patient's perceptions, beliefs and knowledge about cancer on treatment decision making in Pakistan. Asian Pacific Journal of Cancer Prevention: APJCP, 11, 251-255.

[7] Bhurgri, Y., Bhurgri, A., Hassan, S.H., Zaidi, S., Rahim, A., Sankaranarayanan, R. and Parkin, D.M. (2000), Cancer incidence in Karachi, Pakistan: First results from Karachi Cancer Registry. International Journal of Cancer, 85, 325-329.

doi:10.1002/(SICI)1097-0215(20000201)85:3<325::AIDIJC5>3.0.CO;2-J

[8] Considine, J., Livingston, P., Bucknall, T. and Botti, M. (2008) A review of the role of emergency nurses in management of chemotherapy-related complications. Clinical Nursing, 18, 2649-2655.

[9] Verity, R., Wiseman, T., Ream, E., Alderman, E. and Richardson, A. (2008) Exploring the work of nurses who administer chemotherapy. European of Oncology Nursing, 12, 244-252.

[10] McCarthy, E.P., Iezzoni, L.I., Davis, R.B., Palmer, R.H., Cahalane, M., Hamel, M.B. and Davies, D.T. (2000) Does clinical evidence support ICD-9-CM diagnosis coding of complications? http://www.jstor.org/stable/3766966

[11] Barker, K.N., Flynn, E.A., Pepper, G.A., Bates, D.W. and Mikael, R.L. (2002) Medication errors observed in 36 health care facilities. Archives of Internal Medicine, 162, 1897-1903. doi:10.1001/archinte.162.16.1897

[12] Patterson, E.S., Cook, R.I., Marta, L. and Render, F. (2002) Improving patient safety by identifying side effects from introducing bar coding in medication administration. American Medical Informatics Association, 9, 540-553.

[13] Madden, C. (2006) Undergraduate nursing students' acquisition and retention of CPR knowledge and skills. Nurse Education Today, 26, 218-227. doi:10.1016/j.nedt.2005.10.003

[14] Cheryl, B., Lindeman, R. and King, M.L. (2004) Nurse internship pathway to clinical comfort, confidence, and competency. Journal for Nurses in Staff Development, 20, 27-37. doi:10.1097/00124645-200401000-00006

[15] Chan, S.W., Chien, W.T. and Tso, S. (2009) Evaluating nurses' knowledge, attitude and competency after an education programme on suicide prevention. Nurse Education Today, 3, 1-6.

[16] Foubert, J., Kearney, N., Ouwerkerk, J., Unlenhopp, M. and Vaessen, G. (2005) Knowledge of hematological toxicities amongst European nurses-A learning need assessment. European Journal of Oncology Nursing, 9, 239-247.

[17] Papa, D., Kampitsi, A., Katsaragakis, S., Leventelis, C., Papageorgiou, D. and Papadouri, A. (2010) Assessing Hellenic oncology nurses' knowledge and practice about chemotherapy handling and administration. European Journal of Oncology Nursing, 14, 278-282. doi:10.1016/S1462-3889(10)70116-2

[18] Jones, L. and Coe, P. (2004) Extravasations. European Oncology Nursing Society, 8, 355-358. doi:10.1016/j.ejon.2003.10.003

[19] Yates, P., Aranda, S., Hargraves, M., Mirolo, B., Clavarino, A., McLachlan, S. and Skerman, H. (2005) Randomized controlled trial of an educational intervention for managing fatigue in women receiving adjuvant chemotherapy for early-stage breast cancer. Journal of Clinical Oncology, 23, 6027-6036. doi:10.1200/JCO.2005.01.271

[20] Matt, B. and Vaughn, T.A. (1996) Multi-site study of nurse staffing and patient occurrences. Nursing Economics, 16, 196-203.

[21] Fink, A. (2003) The survey handbook. Sage, London.

[22] Rinke, M.L., Shore, A.D., Morlock, L., Hicks, R.W. and Miller, M.R. (2007) Characteristics of pediatric chemotherapy medication errors in a national error reporting database. Cancer, 110, 186-195. doi:10.1002/cncr.22742

[23] Koceja, V.A. (2003) Handle with care: Nurses as pharmacists vs. nurses as nurses. Cancer Nursing, 14, 10111013.

[24] Mohans, S., Wilkes, L.M., Ogunsiji, O. and Walkera, A. (2005) Caring for patients with cancer in non-specialist wards: The nurse experience. European Journal of Cancer Care, 1, 256-263. doi:10.1111/j.1365-2354.2005.00566.x

[25] Jones, L. and Coe, P. (2004) Extravasations. European Oncology Nursing Society, 8, 355-358. doi:10.1016/j.ejon.2003.10.003

[26] Ung, L., Cook, S., Edwards, B., Hocking, L., Osmond, F. and Buttergieg, H. (2002) Peripheral intravenous Cannulation in nursing: Performance predictors. Infusion Nursing, 25, 189-195.

[27] Corner, J. and Barnett, J.W. (1992) The newly registered nurse and the cancer patient: An educational evaluation. International Journal of Nursing Studies, 29, 177-190. doi:10.1016/0020-7489(92)90007-4 